\title{
Crystal Structure Determination of a Salicylate 1,2- Dioxygenase from Pseudaminobacter salicylatoxidans
}

\author{
I. Matera, M. Ferraroni, S. Bürger ${ }^{1}$, A. Stolz', and F. Briganti \\ Department of Chemistry, University of Florence, Via della Lastruccia 3, 50019 Sesto Fiorentino Florence, Italy \\ ${ }^{1}$ Institut für Mikrobiologie, Universität Stuttgart, Allmandring 31, 70569 Stuttgart, Germany
}

The oxygenolytic cleavage of the aromatic nucleus by bacteria requires in most cases the presence of two hydroxyl groups attached to the aromatic ring. Only a few examples have been previously described in which monohydroxylated aromatic compounds were cleaved by ring fission dioxygenases. Recently a new ring fission dioxygenase from the naphthalenesulfonate-degrading strain Pseudaminobacter salicylatoxidans, which oxidized salicylate by a novel ring fission mechanism to 2-oxohepta-3,5-dienedioic acid has been described [1].<smiles>[R3]c1cc(C(=O)O)c(O)c([R2])c1[R]</smiles>

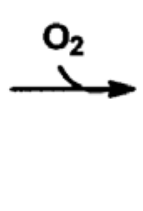<smiles>[R]C(=CC(=O)C(=O)O)C([R2])=C([R])O</smiles>

The salicylate dioxygenase activity from $P$. salicylatoxidans $\mathrm{BN} 12$ is rather unique among the currently known ring fission dioxygenases because the enzyme is able to cleave various substituted salicylates that carry only a single hydroxy group and that are not activated by additional electrondonating substituents for a ring fission reaction. Previous biochemical characterization of the salicylate dioxygenase activity from $P$. salicylatoxidans BN12 demonstrated that the enzyme converted gentisate, 5-aminosalicylate, and 1-hydroxy-2-naphthoate with much higher catalytic activities compared with salicylate and suggested that the ring fission dioxygenase was also structurally similar to gentisate 1,2-dioxygenase or 1-hydroxy-2-naphthoate dioxygenase. This was indicated by the size of the subunits, the structure of the holoenzyme, and the dependence of the enzyme from $\mathrm{Fe}_{2}{ }^{+}$ions. Nevertheless, it became evident that the ring fission dioxygenase from $P$. salicylatoxidans was clearly different from the presently known gentisate 1,2-dioxygenases or 1hydroxy-2-naphthoate dioxygenases because of its unique ability to oxidatively cleave salicylate and also the ability to cleave gentisate and 1-hydroxy-2-naphthoate with high catalytic efficiencies [1].

The enzyme from P. salicylatoxidans BN12 was heterologously expressed in Escherichia coli and purified as a His-tagged enzyme variant. The deduced amino acid sequence encoded a protein with a molecular mass of $41,176 \mathrm{Da}$, which showed 28 and $31 \%$ sequence identity, respectively, to a gentisate 1,2-dioxygenase from Pseudomonas alcaligenes NCIMB 9867 and a 1-hydroxy-2naphthoate 1,2-dioxygenase from Nocardioides sp. KP7 [1].

In order to allow a more detailed analysis of the relationship between the mechanistic capabilities of this particular ring fission dioxygenase and its structural features, this enzyme was crystallized and x-ray diffraction data were collected.

Crystallization experiment were performed using the sitting drop vapor diffusion method. Diffraction quality crystals were obtained at $277 \mathrm{~K}$ from a solution containing $12 \% \mathrm{w} / \mathrm{v} \mathrm{EtOH,} \mathrm{4 \%}$ w/v PEG400, 0.1 M sodium acetate $\mathrm{pH}$ 4.6. Drops were prepared using $1 \mu 1$ protein solution mixed with $1 \mu 1$ reservoir solution and were equilibrated against $100 \mu 1$ precipitant solution [2].

A complete data set at $100 \mathrm{~K}$ extending to a maximum resolution of $2.9 \AA$ was collected at the EMBL beamline BW7B, Hamburg, Germany. Data were collected adding $30 \%$ glycerol to the mother liquor as cryoprotectant, using a MAR345 image plate detector and a wavelength of 0.8423 $\AA$. Crystals belong to the primitive tetragonal space group $\mathrm{P} 4{ }_{3} 2_{1} 2$ with unit cell dimensions $\mathrm{a}=133.3, \mathrm{c}=191.51$. Assuming one tetramer per asymmetric unit the solvent content is $47 \%$ of the unit cell $\left(\mathrm{Vm}=2.3 \mathrm{~A}^{3} / \mathrm{Da}\right)$. Data processing with Mosflm and SCALA gave 38218 unique reflections, an $\mathrm{R}_{\text {symm }}$ of $12.1 \%$ and an overall completeness of $99.0 \%$. Attempts were made in order to solve the structure of the enzyme by a MAD experiment using the anomalous signal of the catalytic Fe(II). From these experiments resulted that the iron content in the native protein was too 
low and prevented us to solve the structure by this technique. Molecular replacement using coordinates of an extradiol dioxygenase structure recentely solved, gentisate 1,2-dioxygenase from Escherichia coli O157:H7 (PDB accession code 2D40) [3], as a model succeeded in finding a solution for salicylate 1,2 dioxygenase. The two enzymes are both homotetramer and a sequence comparison between the two monomeric subunits shows a relatively low sequence identity $(33.3 \%)$. Refinement of the model using the program Refmac is still under work. In the active site the Fe(II) ion is coordinated by three histidines (His119, His121, His160) and at least one water molecule. The detailed analysis of the structural data is underway.

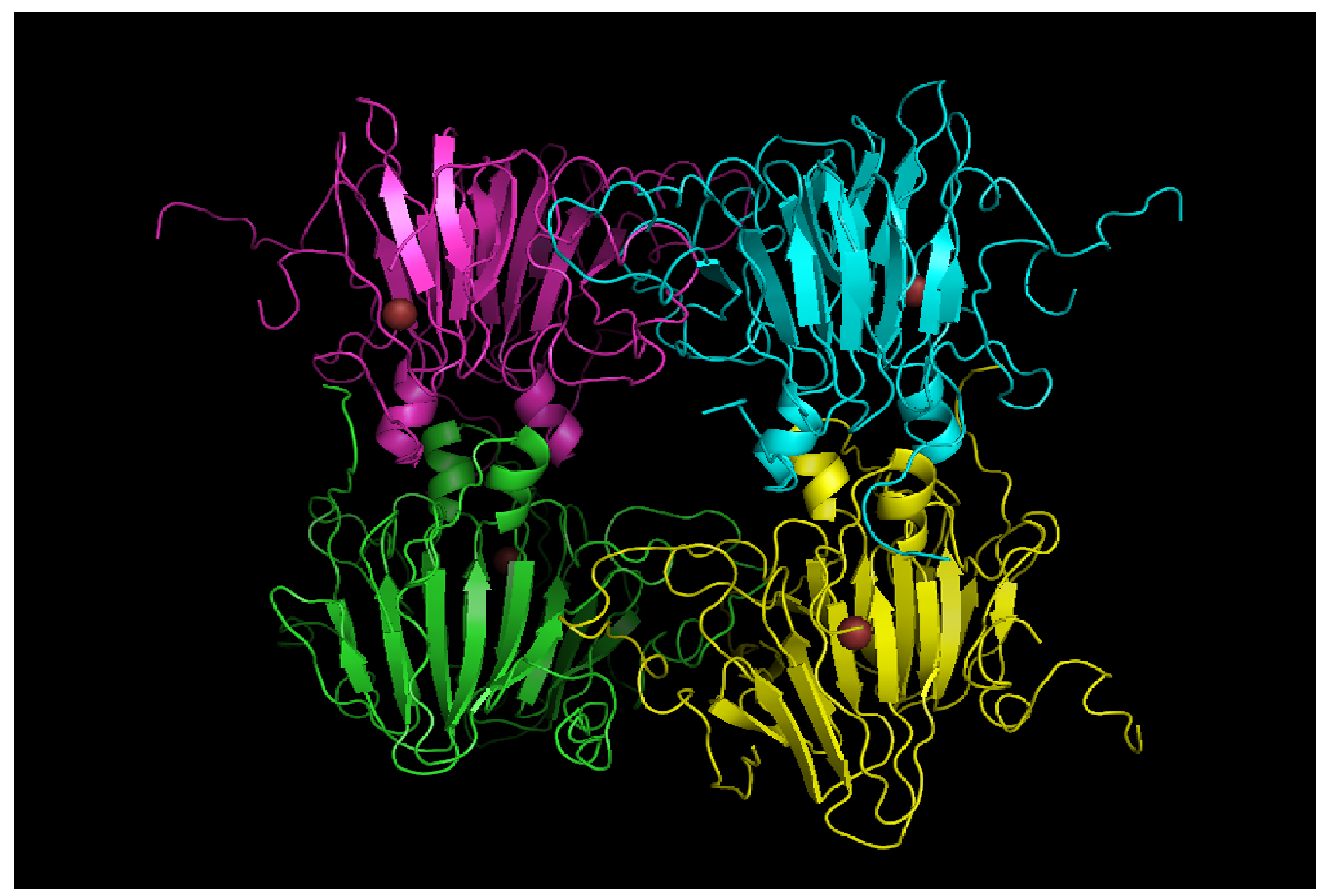

Figure 1: Crystallographic model of salycilate 1,2-dioxygenase from Pseudaminobacter salicylatoxidans

\section{References}

[1] J. P. Hintner, T. Reemtsma, A. Stolz, Journal of Biological Chemistry, 279, 37250 (2004)

[2] I. Matera, M. Ferraroni, S. Burger, A. Stolz, F. Briganti, Acta Cryst. Section F 62, 553 (2006)

[3] M. A. Adams, V. K. Singh, B. O. Keller, Z. Jia, Molecular Microbiology 61, 1469 (2006) 